\title{
Physical properties of a laser beam and the intracavity quantum state
}

David T. Pegg

Centre for Quantum Dynamics, School of Biomolecular and Physical Sciences, Griffith University, Nathan, Brisbane Q 4111, Australia

\begin{abstract}
We use the impossibility of sending information faster than light to show that it is impossible to distinguish between various types of laser cavity field state, such as a Fock state of uncertain energy and any superposition of Fock states of unknown free evolution time, by means of the physically measurable properties of the laser beam. Superpositions can include coherent states, squeezed states and cat states, indeed any pure intracavity state will produce the characteristic properties of the beam such as intrinsic phase coherence.
\end{abstract}

Keywords: Laser beam, coherent states, phase coherence.

Corresponding author: Prof. D. T. Pegg, Centre for Quantum Dynamics, School of Biomolecular and Physical Sciences, Griffith University, Nathan, Brisbane Q 4111, Australia

Phone: + 61733413589 Fax: + 61737354426

E-mail address: David.Thomas.Pegg@gmail.com 


\section{Introduction}

The state of a beam of light emitted by a cavity containing a field in a pure photon number, or Fock, state will be very different from that of a beam emitted if the cavity field is in a coherent state. The intrinsic phase coherence of a laboratory laser beam is well explained if the beam is considered to be in a coherent state, which implies that the intracavity field must also be in a coherent state. If a beam in a coherent state is divided into separate successive packets then each packet will be in a coherent state and all the packets will have a well-defined phase relationship with each other for times shorter than the phase diffusion time. All experimental evidence so far supports the widely held belief that the laser beam is in a coherent state resulting from an intracavity field in a coherent state, including an early experiment by Pfleegor and Mandel [1] showing interference between two laser beams. A coherent state beam has attractive properties in addition to phase coherence, including not being entangled with the intracavity field and being able to be treated as a classical field in many circumstances. It came, therefore, as somewhat of a surprise when Mølmer [2,3] challenged the conventional wisdom concerning the state of a laser beam by using a simple model of light leaking from a cavity. He showed that light beams from cavities containing pure Fock states give similar balanced homodyne interference signals to those from cavities containing coherent states. Examination of the way in which the intracavity state is generated in a typical laboratory laser led him to the conclusion that the use of coherent states to describe laser light is merely a convenient fiction. Mølmer [3] stated that, for large photon numbers, the Poisson distribution of a coherent state is so narrow that the average over different photon number state components equals the result obtained with just one characteristic choice of 
a pure number state equal to the mean of the distribution; so even single pure Fock states must yield the same interference phenomena as pure coherent states.

Not surprisingly, Mølmer’s work stimulated a controversial debate on the subject [4-11] and the issue is not yet settled. The preferred ensemble fallacy, that is, that there can be no privileged partitioning of a density operator [12], has been invoked in support of Mølmer's view [5]. This implies that the intracavity density operator can represent either a mixture of coherent states or a mixture of photon number states and any choice is a matter of convenience only. The fallacy is based on the notion that within the framework of standard of quantum mechanics the density operator is the complete description of the quantum state. By considering phenomena other than interference, however, defenders of the reality of coherent states as a description of laser light have maintained that there are good physical reasons, as opposed to mathematical convenience, for using a coherent state description. Van Enk and Fuchs [6] pointed out that the arguments involving the preferred ensemble fallacy are based on an application of the standard description of the laser field inside the cavity whereas the relevant properties are those of the light outside the cavity. They give a formulation, involving the quantum de Finetti theorem, showing why the coherent state plays a privileged and unique role in the description of propagating laser fields. Noh and Carmichael [11] proposed that an important property for establishing the laser state as a coherent state is disentanglement of the light from its target qubit and in this respect coherent states have some unique properties.

If the external light can be determined by some experimental means to be in a coherent state, then the intracavity field must also be in a coherent state and the question 
would be settled. The assignment of a coherent state would then no longer be merely a convenience as Mølmer suggests. The question then is whether or not there are physical properties of the external laser beam that depend on whether the intracavity state is a pure Fock state, for example, or a coherent state. The mathematical description of coherent states are very different from those of Fock states, indeed the former are sometimes described as classical states with the latter being described as quantum states, so one might expect that there should be some physical properties of a beam that can be used to distinguish between an intracavity coherent state and an intracavity Fock state. As pointed out by van Enk and Fuchs [6], an obvious property of an intracavity coherent state is the production of a beam with intrinsic phase coherence, that is, with different packets having a well-defined phase relationship with each other for a time less than the phase diffusion time. Further, on this time scale each packet is in the same coherent state. In principle at least, one should be able to reconstruct the intracavity coherent state from measurements done on the individual packets. At first sight it may seem unlikely that an intracavity Fock state, which has a uniform phase distribution, can produce a beam with precisely the same phase coherence. This was the situation investigated in Ref. [13] by explicit calculation of the intrinsic phase coherence of a beam obtained from a strong intracavity Fock state by means of a partially reflecting mirror. Although the beam had no particular phase it was found to have the same intrinsic phase coherence as a coherent state. Even the observed phase diffusion effect [13,14] was recovered.

Although strong intracavity Fock states seem to produce external beams with some properties similar to those of beams produced by strong intracavity coherent states, which is consistent with Mølmer's assertion above, perhaps it still may be possible to 
distinguish between coherent and photon number intracavity states if they are weak enough. It should be possible to adopt the approach of Ref. [13] for light leaking from a cavity without making the large number state approximation to examine this question, but it is more useful to adopt a more general approach that can be applied to any measurable property of the beam. We do so in this paper, where we examine the question as to how much the characteristic properties of a beam leaking from a cavity actually depend on the type of intracavity state.

\section{Dependence of beam properties on the cavity state}

To determine whether different beams have different physical properties that can be used to distinguish between types of intracavity states, we consider the case where Alice and Bob are spatially separated and share an entangled state which at a particular time $t_{0}$ is given by

$$
|f\rangle=\sum_{n} c_{n}|n\rangle_{A}|n\rangle_{B}
$$

where $n$ is the photon number and the subscripts refer to fields held by Alice and Bob in their own separate perfect cavities. Both Alice and Bob know the coefficients $c_{n}$. With the prior agreement of Alice, Bob makes either a photon number or a phase measurement at this time but does not tell Alice which measurement is made or the outcome. Alice then replaces one of the perfectly reflecting mirrors of her cavity with a partially transmitting mirror and examines the beam of light slowly leaking out. We assume that the separation between Alice and Bob is sufficient for Alice to complete her experiments on her beam before a signal from Bob sent at $t_{0}$ can reach her. 
If Bob makes a photon number measurement, with outcome $m$ photons, the state inside Alice's cavity becomes the projection of the entangled state $|f\rangle$ onto the number state $|m\rangle_{B}$, that is, it becomes the number state $|m\rangle_{A}$. A phase measurement can be represented by a positive operator valued measure [15,16] with elements $|\varphi\rangle_{B \text { B }}\langle\varphi| d \varphi$ where the phase state

$$
|\varphi\rangle_{B}=(2 \pi)^{-1 / 2} \sum_{n} \exp (-\operatorname{in} \varphi)|n\rangle_{B} .
$$

Thus, if Bob makes a phase measurement with outcome $\theta$ instead of a photon number measurement, the state inside Alice's cavity becomes the projection of $|f\rangle$ onto $|\theta\rangle_{B}$, that is, the superposition state

$$
\begin{aligned}
|\alpha(\theta)\rangle & =\sum_{n} c_{n} \exp (\operatorname{in} \theta)|n\rangle_{A} \\
& =\exp \left(i \hat{N}_{A} \theta\right) \sum_{n} c_{n}|n\rangle_{A}
\end{aligned}
$$

where $\hat{N}_{A}$ is the photon number operator acting on the field in Alice's cavity. If there were any measurement of any physical property that Alice could make on either her intracavity field or on the beam of light emitted from the cavity that would distinguish the photon number state $|m\rangle_{A}$ from the superposition state $|\alpha(\theta)\rangle$, or that would even partially favour one of these, then Bob could send her information at a speed greater than that of light. These states must therefore be completely indistinguishable by Alice by means of any physical measurement.

As the coefficients $c_{n}$ are known, in the absence of a knowledge of $\theta,|\alpha(\theta)\rangle$ can be described as a known superposition state with an unknown phase shift $\theta$ or 
equivalently, by writing $\theta=\omega t$ where $\omega$ is the frequency of the light, as a known superposition state of unknown free evolution time $t$. In the special case where the superposition state is a coherent state, we see that the external beam must have the same measurable physical properties whether the intracavity field is in a coherent state of appropriate phase or a photon number state of appropriate intensity. For example if we divide the external beam into packets, we must find the intrinsic phase coherence discussed above. This applies for all intracavity states $|\alpha(\theta)\rangle$, whether they are coherent states or quantum states such as squeezed states or Schrödinger cat states. It is impossible to distinguish between an intracavity Fock state of unknown photon number and any superposition state of unknown evolution time by any experiments performed on the cavity field itself, on the external beam or on packets of the external beam. As such experiments include interference experiments, we see that our result justifies and strengthens Mølmer’s statement above. It goes further than this, however, as the indistinguishability is not restricted to strong Fock and coherent states but applies to all Fock and superposition states including weak states. An intracavity field in any pure state, even if it does not have a well-defined phase, will produce a beam with observable properties, such as intrinsic phase coherence, that are characteristic of a laser beam.

Our result is also consistent with the preferred ensemble fallacy. Indeed it is possible to use our result to derive the fallacy for this case. In the absence of any information from Bob, Alice would describe her state by $\hat{\rho}_{A}$, the trace of the density operator $|f\rangle\langle f|$ over Bob's cavity states. It is not difficult to show that this can be expressed as 


$$
\begin{aligned}
\hat{\rho}_{A} & =\sum_{n}\left|c_{n}\right|^{2}|n\rangle_{A A}\langle n| \\
& =(2 \pi)^{-1} \int_{0}^{2 \pi}|\alpha(\theta)\rangle\langle\alpha(\theta)| d \theta .
\end{aligned}
$$

This density operator would describe both a mixture of Fock states and a mixture of superposition states $|\alpha(\theta)\rangle$. The fact that she has no experimental basis for distinguishing between the Fock states and the superposition states implies that she has no reason, apart from mathematical convenience, for preferring one description over the other as far as predicting the results of experiments is concerned.

\section{Discussion and conclusion}

The above arguments show that a unique partitioning, or decomposition, of the density operator for the intracavity field cannot be determined from the results of measurements on this field or on the beam. The density operator itself is all that is needed to predict the results of such measurements and it is a fallacy to assume that any particular decomposition will predict physically measurable properties of the intracavity field or the beam that other decompositions will not. We should be cautious, however, before using the preferred ensemble fallacy for purposes other than predicting properties of the system described by the density operator. For example we should not conclude that the density matrix is the best possible quantum description of a mixture of pure states for all situations, that is, to conclude that there is never any situation in which a more accurate quantum description is given by a particular decomposition. Consider a situation similar to the above but in which Alice retains the field in her perfect cavity until she receives a message from Bob, who informs her that he has performed a photon 
number measurement but does not inform her of the outcome. The density operator that she would then use to describe her field would be $\hat{\rho}_{A}$, just as for the previous situation. Now, however, a better quantum description of her field, in that it also contains the information received from Bob, would specify the set of states $|n\rangle_{A A}\langle n|$ with the associated values of $\left|c_{n}\right|^{2}$, which in this case are the probabilities that the states $|n\rangle_{A \text { A }}\langle n|$ were prepared as a result of Bob’s measurement. It would now be justifiable for Alice to describe this field as being in a Fock state of unknown number and not in a superposition state of unknown free evolution time as the latter description would not be consistent with the preparation procedure that includes Bob’s measurement. Although the latter description is just as good as the former in enabling Alice to calculate the outcome probabilities for future measurements on her field, the former description also enables her to retrodict the outcome of Bob’s measurement by choosing to make a photon number measurement on her field. Her retrodiction could be verified by Bob. This is a situation where a particular decomposition can be used to determine a verifiable result that cannot be found from knowledge of the density operator alone and which thus might be said, with some justification, to provide a better quantum description than the density operator. The question now is whether or not a similar argument can be applied to a laboratory laser in order to assign a preferred decomposition on grounds other than convenience. In a laboratory laser it is most likely that the intracavity light and the atoms of the laser medium are in an entangled state $[2,3]$, so a suitable measurement on the atoms could in principle prepare the light in a mixed state described by a particular decomposition of the density operator. However such a measurement is normally not done, in which case there 
is no justification in assigning a particular decomposition such as a coherent state on this basis.

In conclusion, we have re-examined the question of the state of a light emitted by a cavity from the point view of the impossibility of sending information faster than light. We have arrived at a stronger conclusion than that of Mølmer [2,3] who stated that strong intracavity single pure Fock states must yield the same interference phenomena as pure coherent states. We find that, without restriction to strong states, all physical properties of the beam from an intracavity field in a Fock state of uncertain energy but with a known number state probability distribution must be indistinguishable from those from an intracavity state in a known superposition state but of unknown free evolution time. The latter includes coherent states of unknown mean phase and also quantum states such as squeezed or cat states. This approach offers additional insight to one based on the preferred ensemble fallacy but leads to the same conclusion that there is no reason, other than convenience, for preferring a coherent state description of a laboratory laser beam in order to determine its measurable properties or the outcome of experiments done with it.

\section{References}

[1] R. L. Pfleegor and L. Mandel, Phys. Lett. 24A (1967) 766.

[2] K. Mølmer, Phys. Rev. A 55 (1997) 3195.

[3] K. Mølmer, J. Mod. Opt. 44 (1997) 1937.

[4] J. Gea-Banacloche, Phys. Rev. A 58 (1998) 4244.

[5] T. Rudolph and B. C. Sanders, Phys. Rev. Lett. 87 (2001) 077903.

[6] S. J. van Enk, and C. A. Fuchs, Phys. Rev. Lett., 88 (2002) 027902. 
[7] B. C. Sanders, S. D. Bartlett, T. Rudolph, and P. L. Knight Phys. Rev. A 68 (2003) 042329.

[8] H. M. Wiseman, J. Opt. B: Quantum Semiclass. Opt. 6 (2004) S849.

[9] H. Cable, P. L. Knight, and T. Rudolph, Phys. Rev. A 71 (2005) 042107.

[10] D. T. Pegg and J. Jeffers: J. Mod. Opt. 52 (2005) 1835.

[11] C. Noh and H. J. Carmichael, Phys. Rev. Lett., 100 (2008) 120405.

[12] P. Kok and S. L. Braunstein Phys. Rev. A 61 (2000) 042304.

[13] D. T. Pegg, Phys. Rev. A 79 (2009) 053837.

[14] S. M. Barnett, S. Stenholm and D. T. Pegg, Opt. Commun. 73 (1989) 314.

[15] C. W. Helstrom, Quantum Detection and Estimation Theory, Academic Press, New York, 1976.

[16] J. A.Vaccaro and D. T. Pegg, Physica Scripta T48 (1993) 22.

Corresponding author: David T. Pegg 